\section{Into the fire}

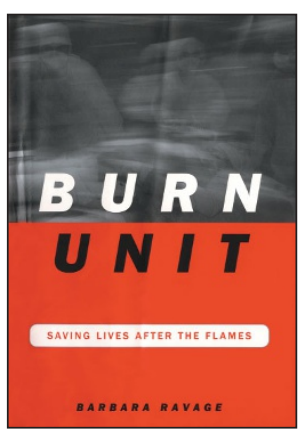

\section{Burn Unit: Saving Lives After the Flames}

by Barbara Savage

Da Capo Press, 2004

288 pp. hardcover, $\$ 26$

ISBN 0306813467

\section{Reviewed by Steven E Wolf}

It was a pleasure to review Barbara Ravage's Burn Unit, which is a study of the people, environment and care administered in the Massachusetts General Burn Unit. Although this comprehensive study was done at a major burn unit in Boston, it could have reflected events in any of several burn units across the country. It was almost eerie to read Ravage's characterization of the personnel and how they did their jobs. I thought, "I know someone like that in my unit." She describes the responses of the patients and their families to the life-changing event of a major burn very accurately and in a nonthreatening but realistic fashion. In particular, human resilience in the face of adversity, something those of us in the burn field encounter daily, was fairly portrayed.

Ravage chronicles the initial care, treatment and travails associated with recovery in two burned patients. Along the way, we meet the surgeons, nurses, therapists, social workers, dietitians, burn technicians and others who provide care and treatment, as well as the families of those who have been burned. She also does a fine job describing the history of burn care as a foundation for her description of modern burn care. As part of this history, Ravage provides a blow-by-blow account of the fire at the Cocoanut Grove nightclub in Boston during the World War II, which killed 492 people and was a turning point for burn care worldwide. The description is informative even to the expert, and it sets the stage for the rest of the book. The personalities who would go on to make some of the breakthroughs in burn care, such as Jack Burke and his role in early wound excision are also justly described.

The changes that occur after a burn and the ravages of healing are covered in the next chapter-an absolute delight to read, in clear, vivid language such as is all too rare in medical writing. The language clinicians speak to one another is oftentimes sterile,

Steven E. Wolf is in the Department of Surgery, University of Texas Health Science Center-San Antonio, and at the Burn Center, Brooke Army Medical Center, US Army Institute of Surgical Research, San Antonio, Texas 78234, USA. cumbersome and incomprehensible (evident, for instance, in any episode of the TV series ER); I, for one, will refer to this book for these descriptions when searching for clarity in explaining burn care.

Later in the book we meet two burn victims and their families. Ravage describes the events surrounding their particular injuries and the effects it had on them at a personal level, which lets the reader understand their world, and by proxy that of any person with a devastating burn. We meet the real, downto-earth professionals involved in their care. Ravage does not glorify the people providing this care, and the requirements and quirks of the job are accurately represented at a personal and nondetached level, which is where burn practitioners live. We are, after all, people who have to deal with these devastating problems, which are abhorrent to most at first glance. Ravage makes it real and understandable, which was a great comfort to read and, might I add, right on the mark. Also, most of the actual care administered in medicine is not necessarily done by doctors, even though we get most of the credit. Ravage does a wonderful job of highlighting the involvement of non-physicians in this book.

In her discussion, Ravage takes us through the initial resuscitation, wound care, associated problems with the lungs, skin grafting, and early rehabilitation. The reader can understand how the body reacts to a burn at a biological level, from the initial injury to the healing process and eventual scarring. But again, Ravage brings us back to the people, letting us know what it is like to be a burn care practitioner or a patient or family member.

My only quibble with the book was the focus on some of the treatments done at Massachusetts General that are neither common practice nor, in my opinion, necessary (such as the use of individual isolation beds in the intensive care unit, or hyperbaric treatments), but this is a personal pet peeve. Ravage ends with another vivid description of wound healing and the quest for a skin product that will provide wound coverage and decrease scarring, a major research goal that has not yet been achieved satisfactorily.

One of the central themes of Burn Unit emerges in Ravage's own words, "The truth about burns is that easy answers are in short supply." This could not be more correct. The book addresses this statement in many ways, and is quite comprehensive in doing so. One of the clear strengths of the book is the manner in which burns and their care are described in an eminently readable form to anyone, regardless of training or background. The author's descriptions are palpable and real, and lay a more-than-adequate foundation for explaining the basis of burn care. I can recommend the book without reservation to anyone who is interested in burned people, particularly those in the medical field but also patients, families of burned patients, and any who are interested in the environment and culture of the quirky, sometimes lonely, and often socially abandoned burn unit. 